\title{
Use of a biological reactor and platelet-rich plasma for the construction of tissue-engineered bone to repair articular cartilage defects
}

\author{
HUIBO LI ${ }^{1}$, SHUI SUN ${ }^{2}$, HAILI LIU ${ }^{2}$, HUA CHEN $^{1}$, XIN RONG $^{1}$, JIGANG LOU $^{1}$, \\ YUNBEI YANG $^{1}$, YI YANG ${ }^{1}$ and $\mathrm{HAO} \mathrm{LIU}^{1}$ \\ ${ }^{1}$ Department of Orthopedics, West China Hospital, Sichuan University, Chengdu, Sichuan 610041; \\ ${ }^{2}$ Department of Orthopedics, Shandong Provincial Hospital Affiliated to Shandong University, \\ Jinan, Shandong 250021, P.R. China
}

Received May 25, 2015; Accepted April 14, 2016

DOI: 10.3892/etm.2016.3380

\begin{abstract}
Articular cartilage defects are a major clinical burden worldwide. Current methods to repair bone defects include bone autografts, allografts and external fixation. In recent years, the repair of bone defects by tissue engineering has emerged as a promising approach. The present study aimed to assess a novel method using a biological reactor with platelet-rich plasma to construct tissue-engineered bone. Beagle bone marrow mesenchymal stem cells (BMSCs) were isolated and differentiated into osteoblasts and chondroblasts using platelet-rich plasma and tricalcium phosphate scaffolds cultured in a bioreactor for 3 weeks. The cell scaffold composites were examined by scanning electron microscopy (SEM) and implanted into beagles with articular cartilage defects. The expression of osteogenic markers, alkaline phosphatase and bone $\gamma$-carboxyglutamate protein (BGLAP) were assessed using polymerase chain reaction after 3 months. Articular cartilage specimens were observed histologically. Adhesion and distribution of BMSCs on the $\beta$-tricalcium phosphate $(\beta$-TCP) scaffold were confirmed by SEM. Histological examination revealed that in vivo bone defects were largely repaired 12 weeks following implantation. The expression levels of alkaline phosphatase (ALP) and BGLAP in the experimental groups were significantly elevated compared with the negative controls. BMSCs may be optimum seed cells for tissue engineering in bone repair. Platelet-rich plasma (PRP) provides a rich source of cytokines to promote BMSC function. The $\beta$-TCP scaffold is advantageous for tissue engineering due to its biocompatibility and 3D structure that promotes cell
\end{abstract}

Correspondence to: Professor Hao Liu, Department of Orthopedics, West China Hospital, Sichuan University, 37 Guoxue Road, Chengdu, Sichuan 610041, P.R. China E-mail: liuhao6304@163.com

Key words: bone repair, bone tissue engineering, bone regeneration, bioreactor, platelet-rich plasma adhesion, growth and differentiation. The tissue-engineered bone was constructed in a bioreactor using BMSCs, $\beta$-TCP scaffolds and PRP and displayed appropriate morphology and biological function. The present study provides an efficient method for the generation of tissue-engineered bone for cartilage repair, compared with previously used methods.

\section{Introduction}

Bone defects are frequently observed and are a significant issue in clinical practice. The repair of a bone with extensive defects is a complex procedure. Massive bone defects therefore constitute a major challenge in reconstructive surgery. Currently, methods used to repair bone defects include bone autografts, allografts and external fixation (1).

Although there are several ways to repair articular cartilage defects, damage caused by degeneration of articular cartilage, trauma and sport-associated injuries does not regenerate (2). The rapid development of tissue engineering techniques has enabled cartilage and bone regeneration using scaffold material and stem cells. A previous study has focused on the role of single growth factors in bone tissue engineering (3). However, the synergistic effects between various growth factors have rarely been reported (4).

In the present study, beagle bone marrow mesenchymal stem cells (BMSCs) were used as seed cells. Once induced, BMSCs differentiate into osteoblasts and chondroblasts (5). BMSCs were first identified by Fridenshtein (6) and are considered to be optimal stem cells for the clinical treatment of bone and cartilage defects, and have received widespread attention due to their abundance, ease of access, vigorous growth and multi-lineage differentiation (7). The present study used platelet-rich plasma (PRP) as a source of growth factors. PRP was generated by the resuspension of platelets derived from autologous blood at a high concentration in a low volume of plasma. The platelet count in PRP varies according to the preparation technique, ranging from two- to several-fold increases above physiological levels (8). Activation of platelets in PRP by an agonist, such as thrombin, leads to the release of numerous growth factors and other 
important proteins, including basic fibroblast growth factor, fibronectin, insulin-like growth factor-1 (IGF-1), osteocalcin, platelet-derived growth factor, serotonin, transforming growth factor-b1, thrombospondin-1, vascular endothelial growth factor and Von-Willebrand factor (9). A previous study revealed that the application of a single growth factor was not sufficient to achieve the desired effect on BMSCs (10). The aforementioned proteins and growth factors have confirmed efficacy in the osteogenic induction in BMSCs (11). Several of these proteins are involved in wound healing and tissue regeneration (12-15).

Tissue engineering scaffolds are 3D substrates that provide the structural microenvironment required for the cultivation of BMSCs (14). Additionally, tissue engineering scaffolds are used in drug delivery, absorbable sutures, surgical orthopedic devices and cell tissue engineering applications (15). Porous bioceramics, such as $\beta$-tricalcium phosphate $(\beta$-TCP), are excellent scaffolds for tissue engineering; they are easy to combine with BMSCs, and are suitable for the repair of bone defects in articular cartilage injury (16). In the present study, BMSCs and $\beta$-TCP scaffolds were used in a perfusion bioreactor to simulate in vivo conditions that promote cell adhesion, proliferation and differentiation. The current study may present a novel platform for the generation of tissue-engineered bone that is suitable in bone-repair applications.

\section{Materials and methods}

Animal studies. All animal experimental protocols were approved by the local Institutional Animal Care and Use Committee of Shandong University (Jinan, China) in compliance with the 'Guide for the Care and Use of Laboratory Animals' published by the National Academy Press (NIH Publication No. 85-23, revised 1996). Male beagles $(n=10$; 4-10 months of age), weighing between 8 and $10 \mathrm{~kg}$, were obtained from the Experimental Animal Center of Shandong Province (Jinan, China) and used in the present study. Animals were kept in separate cages with an ambient temperature of $24^{\circ} \mathrm{C}$ and $45 \%$ humidity, with a $14 \mathrm{~h}$ light/dark cycle, were fed a standard diet with ad libitum access to food and water, and were allowed to move freely during the study.

Isolation and cultivation of beagle BMSCs. Following the administration of $3 \%$ pentobarbital sodium $(1 \mathrm{ml} / \mathrm{kg}$; Solarbio Science \& Technology Co., Ltd., Beijing, China), the tibia was shaved and treated with $1 \%$ povidone iodine solution (Shandong Luxi Pharmaceutical Co., Ltd., Heze, China). Bone marrow $(5 \mathrm{ml})$ was aspirated with a sterile bone marrow aspiration needle attached to a $10 \mathrm{ml}$ syringe containing $1 \mathrm{ml}$ heparin solution (Shandong Lukang Pharmaceutical Group, Jining, China). Following the addition of an equal volume $(5 \mathrm{ml})$ of phosphate-buffered saline (PBS) to the bone marrow serum, the mixture was homogenized. The aspirate was centrifuged at a speed of $168 \mathrm{x} \mathrm{g}$ for $5 \mathrm{~min}$ at $20^{\circ} \mathrm{C}$. The supernatant was removed and the precipitate mixed with an equal volume of PBS $(5 \mathrm{ml})$ and homogenized again. The mixture was then centrifuged at a speed of $1,048 \mathrm{x}$ g for $20 \mathrm{~min}$ at $20^{\circ} \mathrm{C}$ and the cloudy coat in the centre of the centrifuge tube was harvested. This was combined with $5 \mathrm{ml}$ PBS in a centrifuge tube and separated at a speed of 2,500 rpm for $5 \mathrm{~min}$. Subsequently,
BMSCs were harvested from the bottom of the centrifuge tube. The primary BMSCs were cultured in Dulbecco's Modified Eagle's Medium (DMEM; HyClone, Rockford, IL, USA) supplemented with $10 \%$ fetal bovine serum (Gibco; Thermo Fisher Scientific, Inc., Waltham, MA, USA) at $37^{\circ} \mathrm{C}$, in a humidified incubator containing $5 \% \mathrm{CO}_{2}$. After three passages, the BMSCs were divided into osteoblast- and chondroblast-induced groups. The osteoblast-induced group was cultured in osteogenic differentiation medium containing DMEM supplemented with $10 \%$ fetal bovine serum, $10 \mathrm{nM}$ dexamethasone, $50 \mathrm{mg} / 1$ ascorbic acid and $10 \mathrm{mM}$ $\beta$-glycerophosphate (Wuhan Boster Biological Technology Co., Ltd., Wuhan, China). The chondroblast-induced group was cultured in chondrogenesis medium containing TGF- $\beta 1$ (10 ng/ml), IGF-1 (50 g/l) and dexamethasone (40 g/l).

Cell identification. Cells were observed using a light microscope for morphology and growth status daily. In the chondroblast-induced group, type II collagen was detected by immunohistochemistry. Antibodies were purchased from Wuhan Boster Biological Technology Co., Ltd.. The cells were fixed with $3 \%$ paraformaldehyde for $10 \mathrm{~min}$ and rinsed with Tris buffer, $\mathrm{pH} 7.4$, at room temperature for $5 \mathrm{~min}$. The cells were then incubation with serum $(1: 20$ in Tris buffer) at room temperature for $10 \mathrm{~min}$, incubated with primary antibodies (anti-collagen II; cat. no. BA0533) in a moist chamber overnight at $4^{\circ} \mathrm{C}$, washed with PBS and incubated with biotinylated goat anti-rabbit immunoglobulin $\mathrm{G}$ secondary antibody (1:50 in Tris buffer; cat. no. BA1003) at room temperature for $30 \mathrm{~min}$, washed with PBS and incubated with dual system bridge antibodies (1:50 in Tris buffer; cat. no. BA1003) for $30 \mathrm{~min}$ at room temperature. The cells were then washed with PBS and fuscin (Wuhan Boster Biological Technology Co., Ltd.) was used for staining for $30 \mathrm{~min}$ at room temperature. Subsequently, the specimens were washed with PBS and dried, covered with glycerin/gelatin and examined under a light microscope. In the osteoblast-induced group, cells were identified with alkaline phosphatase (ALP) staining and Alizarin red staining (Wuhan Boster Biological Technology Co., Ltd.) to observe the activity of ALP and the number of mineral nodules.

$3 D$ scaffolds. $\beta$-TCP is a porous bio-ceramic 3D scaffold purchased from Shanghai Bio-lu Biomaterials Co., Ltd. (Shanghai, China). The diameter of the spherical pores was $500-600 \mu \mathrm{m}$ and the porosity was $75 \pm 10 \%$ with $>80 \%$ of the pores being spherical in shape. Using a mold, a cylinder of $\beta$-TCP $5 \mathrm{~mm}$ in diameter and $5 \mathrm{~mm}$ in height was constructed. The surface of the cylinder was smooth and was sterilized using ethylene oxide.

Perfusion bioreactor. The perfusion bioreactor (Fig. 1) was designed by the East China University of Science and Technology (Shanghai, China). The system consists of a peristaltic pump, a device which pulls culture media through silicone tubes, a perfusion column in which the cell-scaffold composite can be implanted, two silicone tubes, an air filter and a flask filled with cell culture media. The function of the two silicone tubes is to enable the flow of culture media into a peristaltic pump via the silicone tubes when the peristaltic pump begins 
to rotate. This creates a circulatory flow to provide mechanical stimulation to the cells.

PRP was prepared according to a two-step centrifugation method as previously described (13). The standard protocol involves the preparation of PRP from autologous blood by a two-step centrifugation process; a separation step and a concentration step $(17,18)$. For the preparation of PRP, $\sim 10 \mathrm{ml}$ of blood was obtained from the femoral veins of 10 anesthetized ( $3 \%$ pentobarbital sodium) beagles. The blood was collected in sterilized tubes containing $1 \mathrm{ml}$ of sodium citrate used as an anticoagulant. The blood was then centrifuged twice; initially at $20 \mathrm{x} \mathrm{g}$ for $16 \mathrm{~min}$ at $20^{\circ} \mathrm{C}$ to remove red blood cells, and then at $1,500 \times \mathrm{g}$ for $12 \mathrm{~min}$ at $20^{\circ} \mathrm{C}$ to obtain the platelet pellet (19). The pellet was resuspended in platelet-poor plasma and the PRP from the 10 beagles was mixed and stored at $-80^{\circ} \mathrm{C}$ until required, and consequently allogenic PRP was used in the subsequent experiments. The PRP was combined with $1 \mathrm{ml}$ coagulant (composed of $1 \mathrm{ml} 10 \% \mathrm{CaCl}_{2}$ and 1,000 U thrombin) and homogenized. Platelet-rich plasma gel, a jelly-like substance (Fig. 2), was then harvested.

Tissue-engineered bone building. Once the cells had been passaged three times, BMSC cell suspensions were prepared by trypsin (Wuhan Boster Biological Technology Co., Ltd.) digestion prior to the addition to the $\beta$-TCP scaffolds. One scaffold was prepared with an osteoblast-induced suspension and another with a chondroblast-induced suspension. Subsequently, $3 \mathrm{ml}$ cell culture media was added to each cultivation orifice plate. The BMSC-TCP composites were placed in an incubator for 2 days at $37^{\circ} \mathrm{C}$ in an atmosphere containing $5 \% \mathrm{CO}_{2}$ (Fig. 3). The composites were then placed in the culture chamber of a perfusion bioreactor with a flow rate of $3.5 \mathrm{ml} / \mathrm{min}$ for 3 weeks using culture media in the presence or absence of PRP. The culture medium was changed every 2-3 days. Adhesion, proliferation and growth of the BMSCs in the $\beta$-TCP scaffold were examined under a scanning electron microscope (SEM). The composites were divided into five groups as follows: i) Group A, composite cultured with PRP in the bioreactor; ii) Group B, composite cultured with PRP without the bioreactor; iii) Group C, composite cultured in the bioreactor without PRP; iv) Group D, composite cultured without PRP and the bioreactor and v) Group E, $\beta$-TCP scaffold only (negative control group).

Surgical procedure. Models of arthrodial cartilage defects were established in 20 joints of 10 beagles, with five defects in each joint. The beagles were anesthetized with an intravenous injection of $3 \%$ pentobarbital sodium. A median incision of $3 \mathrm{~cm}$ was made over the knee joints, soft tissues were dissected and the bone was exposed by gentle retraction of the muscles. Five defects with a depth of $10 \mathrm{~mm}$ and a width of $5 \mathrm{~mm}$ were created in the distal femoral articular surface with a hand brace.

Composite implantation. Once the defects had been irrigated with sterile physiological saline solution, the BMSC-TCP composites were implanted into the defects and press-fitted. The osteoblast-induced BMSC-TCP composite (diameter, $5 \mathrm{~mm}$; height, $5 \mathrm{~mm}$ ) was implanted into the innermost region of the bone defect, and the chondroblast-induced

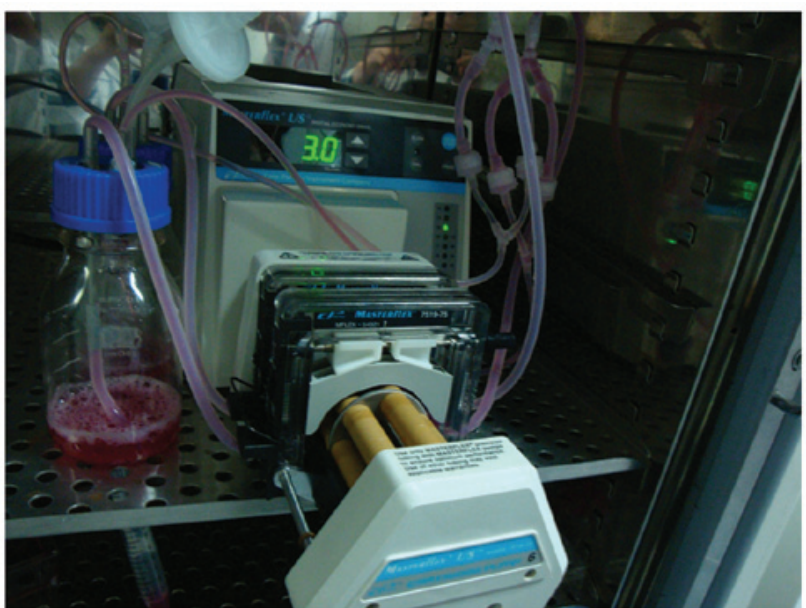

Figure 1. Perfusion bioreactor design used in the present study.

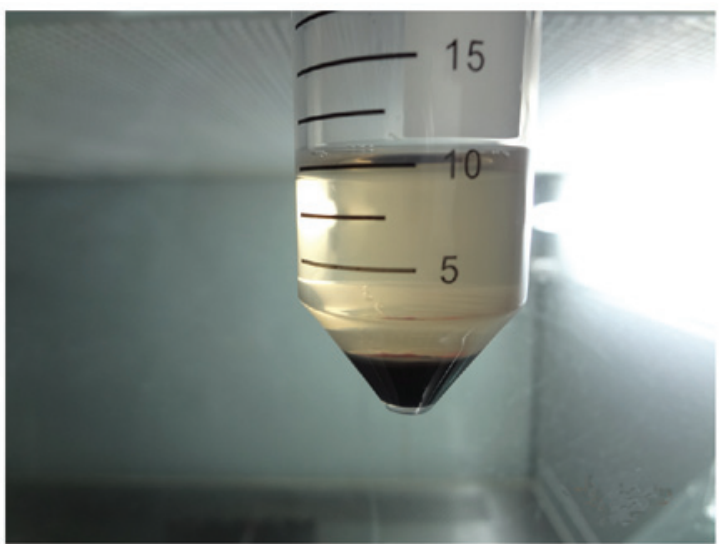

Figure 2. Preparation of platelet-rich plasma

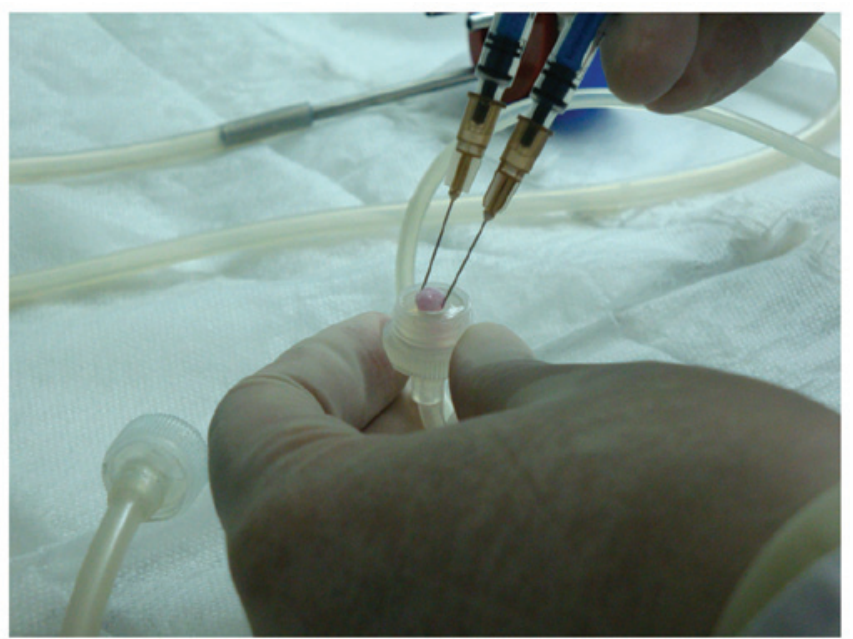

Figure 3. Cell-scaffold placement into the bioreactor.

BMSC-TCP composite of the same dimensions was implanted into the remaining shallow region of the bone defect. The osteoblast-induced BMSC-TCP composite was mechanically forced into contact with the chondroblast-induced composite. Following this, muscles, fascia and skin were separately closed 
over the regions of the defects using 4-0 sterile sutures. The animals were kept in standard conditions and were intramuscularly injected with 800,000 U penicillin (Wuhan Boster Biological Technology Co., Ltd.) each day for 3 consecutive days following the surgical procedure. The animals were then humanely sacrificed by anesthetic overdose $(100 \mathrm{mg} / \mathrm{kg})$ with pentobarbital sodium 3 months following the surgical procedure and the composites with tissue cells nearby were removed in order to perform reverse transcription-quantitative polymerase chain reaction (RT-qPCR) and histological analysis.

Detection of ALP and BGLAP expression levels using $R T-q P C R$. Template RNA used was from the total RNA of the BMSC-TCP composites. Total RNA was isolated using TRIzol reagent (Invitrogen; Thermo Fisher Scientific, Inc.), according to the manufacturer's instructions. The concentration of RNA was determined using an ultraviolet spectrophotometer. QIAGEN RNase-Free DNAse was purchased from Cyagen Biosciences (Santa Clara, CA, USA). The cDNA sample was generated using the RevertAid First Strand cDNA Synthesis kit (Thermo Fisher Scientific, Inc.). Primers sequences used in RT-qPCR were as follows: Forward, 5'-TGTGCGGGG TCAAGGCTAAC-3'; reverse, 5'-GGCGTCCGAGTACCA GTTGC-3' for ALP; forward, 5'-CTCCTTACCCGGATC CCCTG3'; reverse, 5'-GTAGAAGCGCTGGTAGGCGT3' for BGLAP2. Reaction Buffer, RiboLock RNase Inhibitor, dNTP Mix, RevertAid M-MuLV Reverse Transcriptase were provided by Cyagen Biosciences Inc.. A negative control was included. Quantitative RT-PCR was performed using SYBR Green Realtime PCR Master mix (Toyobo, Osaka, Japan) in an Applied Biosystems AB7500 real-time PCR system (Applied Biosystems; Thermo Fisher Scientific, Inc.). Amplification was performed under the following thermal cycling conditions: $95^{\circ} \mathrm{C}$ for $60 \mathrm{sec}$ followed by 40 cycles at $95^{\circ} \mathrm{C}$ for $15 \mathrm{sec}$ and $60^{\circ} \mathrm{C}$ for $60 \mathrm{sec}$. GADPH was used as a reference gene, and the $2^{-\Delta \Delta \mathrm{Cq}}$ method was used (20). RT-qPCR was performed in triplicate and the mean \pm standard deviation was calculated.

Statistical analysis. Data are presented as the mean \pm standard deviation and were analyzed using the SPSS, version 20.0 statistical software package (IBM SPSS, Armonk, NY, USA). One-way analysis of variance or paired Student's t-tests were used for comparisons between groups. $\mathrm{P}<0.05$ was considered to indicate a statistically significant difference.

\section{Results}

Cell culture observation. BMSCs from beagle bone marrow were harvested and characterized microscopically in a cell culture. Following a culture period of 3 days (Fig. 4A), a small number of spindle-shaped primary cells were observed growing against the wall of the cell culture flask. Cell colonies formed after 7 days of culture (Fig. 4B). After 7 days of culture, the cells gathered into swirl-shaped colonies (Fig. 4C).

Characterization of BMSCs. BMSCs were induced to differentiate into chondroblasts for 7 days. The cells displayed brownish yellow cytoplasmic granules following type II collagen immunohistochemical staining (Fig. 5A). Subsequent to 7 days of osteoblastic differentiation, alkaline phosphatase and Alizarin red staining revealed a large quantity of gray-black granules or black deposits (Fig. 5B). The aforementioned observations are consistent with the initial stem cell phenotype, which was further confirmed by the expression of CD29 and CD44 following differentiation. Alizarin red staining revealed a large number of mineral nodes in the osteoblast-induced group (Fig. 5C and D).

Examination of composites by SEM. Differentiated BMSCs were deposited on $\beta$-TCP scaffolds and allowed to grow for 21 days. SEM revealed that cells adhered to the scaffold and were widely distributed, secreting abundant extracellular matrix onto the scaffold (Fig. 6). Cell proliferation and a stretched, extended morphology was also observed, suggesting that the $\beta$-TCP scaffold is a good substrate for BMSC growth (Fig. 6).

Histological analyses of cartilage regeneration in vivo. Once the BMSCs had been cultured for 3 weeks in the bioreactor, BMSC scaffolds (Group A) or controls (Groups B-E) were implanted into an arthrodial cartilage defect model in beagles as described in the Materials and methods section. Histological examination demonstrated that several of the implanted BMSC scaffolds regenerated cartilage tissue, and filled the defective area. Numerous newly differentiated chondrocytes, in addition to abundant bone formation, were observed with many chondrocytes and osteoblasts present. Osteotylus was also observed and this was regularly arranged and dispersed throughout the site of the defect (Fig. 7A).

Several controls (bioreactor, PRP and BMSCs) were performed to investigate the importance of each component of the culture system. Implantation of BMSCs cultured on scaffolds without the bioreactor led to the formation of high levels of hyaline chondrocytes and cartilage lacunae, with the new cartilage integrating with the existing articular cartilage. Conversely, in Group A, new bone was not evenly distributed and the quantity of hyaline chondrocytes was reduced (Fig. 7B). In Group C, in which BMSCs were cultured in the absence of PRP, irregular, newly-formed osteotylus was present in the defect, and chondrocytes and osteoblasts were randomly and loosely arranged (Fig. 7C). In the absence of both PRP and the bioreactor (Group D), a small number of newly-generated chondrocytes and osteoblasts were detected in the defect. New bone formation was minimal, with abundant fibrous tissues infiltrating the damaged area (Fig. 7D). In the negative control with no BMSCs (Group E), several fibroblasts were observed to adhere to adjacent tissue on the defect border, resulting in the proliferation of fibrous and scar tissue (Fig. 7E).

Expression levels of osteogenic differentiation markers in BMSC implants. The expression levels of osteogenic differentiation markers, ALP and BGLAP, in BMSC implants was determined by RT-qPCR (Fig. 8). In experimental Groups A, $\mathrm{B}, \mathrm{C}$ and D, the expression levels of ALP and BGLAP were significantly higher compared with the negative control group (Group E; $\mathrm{P}<0.05$ ). The expression levels of ALP and BGLAP were significantly higher in Group A compared with Group C $(\mathrm{P}<0.05)$ and in Group B compared with Group D $(\mathrm{P}<0.05)$. These results therefore suggested that the presence 
A

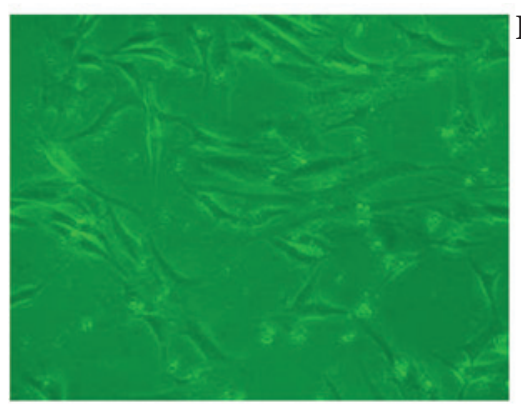

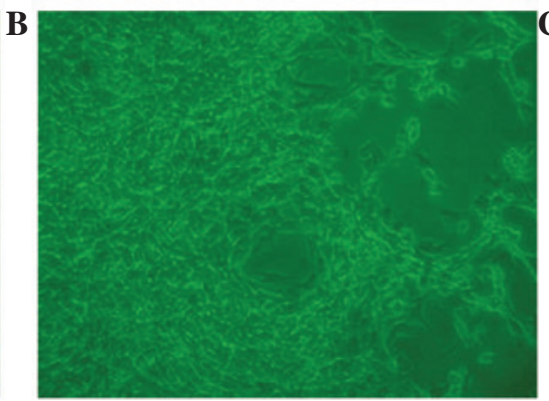

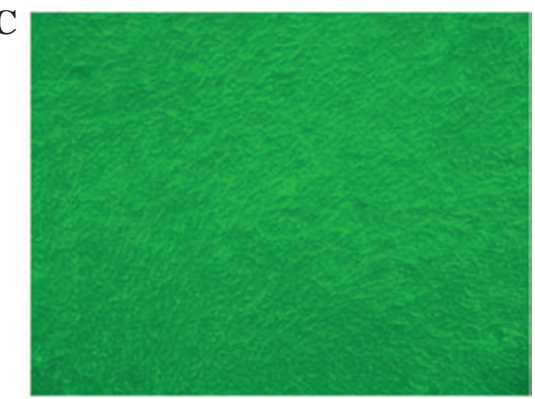

Figure 4. Bone marrow stem cells from beagle bone marrow (A) 3 days after seeding, (B) primary cells cultured for 7 days, and (C) 7 days following subculture (magnification, x100).

A

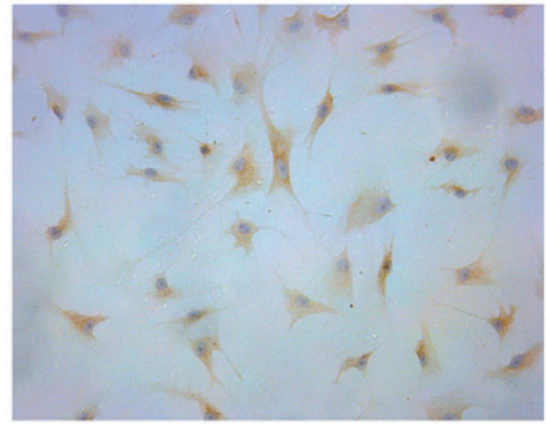

C

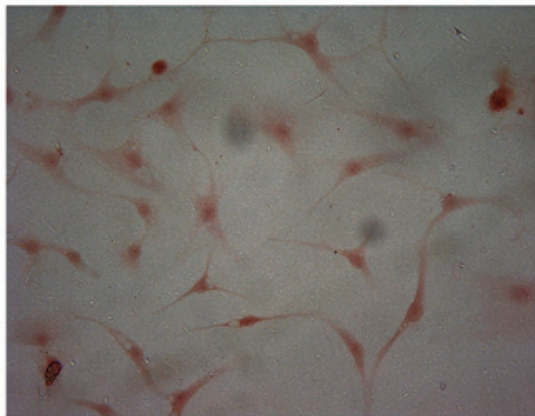

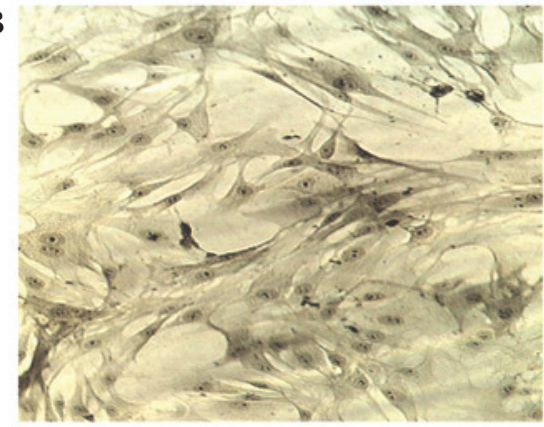

D

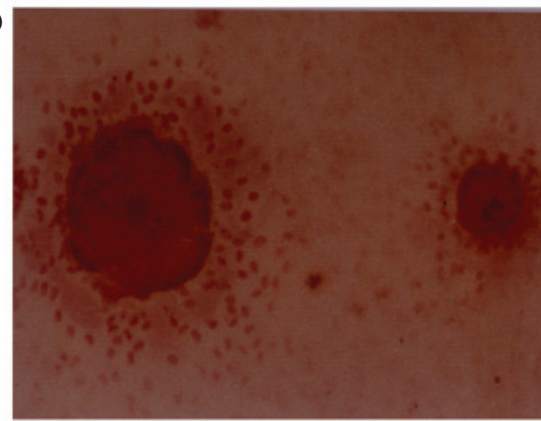

Figure 5. Type II collagen immunohistochemical staining. (A) In the chondroblast-induced group, the cells displayed brownish yellow granules in the cytoplasm following type II collagen immunohistochemical staining (magnification, x200). (B) In the osteoblast-induced group (magnification, x200), gray-black granules or black deposit were observed in the (C) cytoplasm (magnification, x200) and (D) Alizarin red staining revealed a large number of mineral nodes in the osteoblast-induced group (magnification, $\mathrm{x} 400$ ).

A

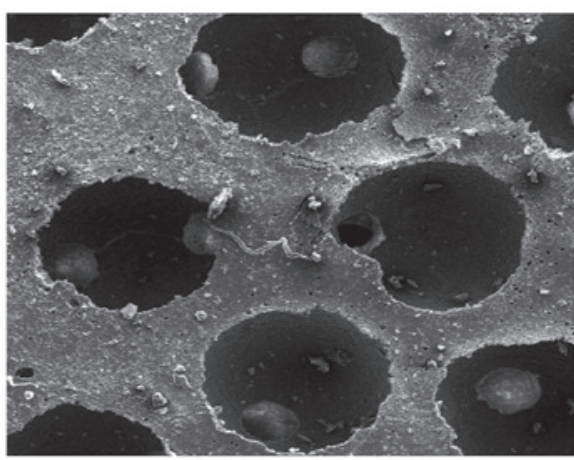

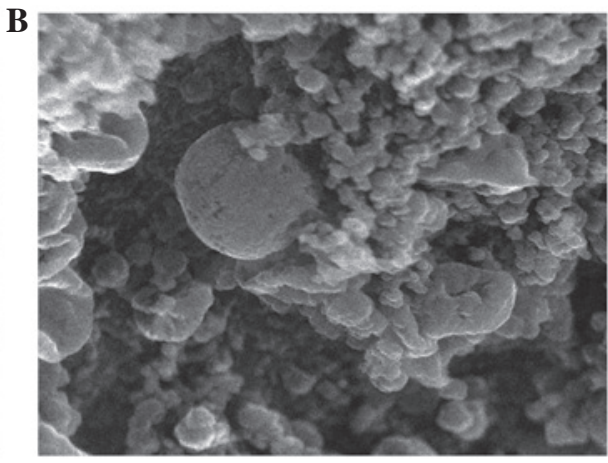

Figure 6. (A) Scanning electron microscopy showed the structure of the $\beta$-tricalcium phosphate scaffold. (B) Scanning electron microscopy revealed good adhesion and distribution of beagle bone marrow mesenchymal stem cells on the $\beta$-tricalcium phosphate scaffold. (magnification, $x 5,000)$.

of PRP promotes bone regeneration. The expression levels of ALP were not significantly higher in Group A compared with Group B, or in Group C compared with Group D, (Group A compared with Group B, $\mathrm{t}=0.169, \mathrm{P}>0.05$; Group C compared with Group $\mathrm{D}, \mathrm{t}=0.646, \mathrm{P}>0.05)$. The present data supports the hypothesis of an osteogenic promoting effect of the bioreactor. 
A

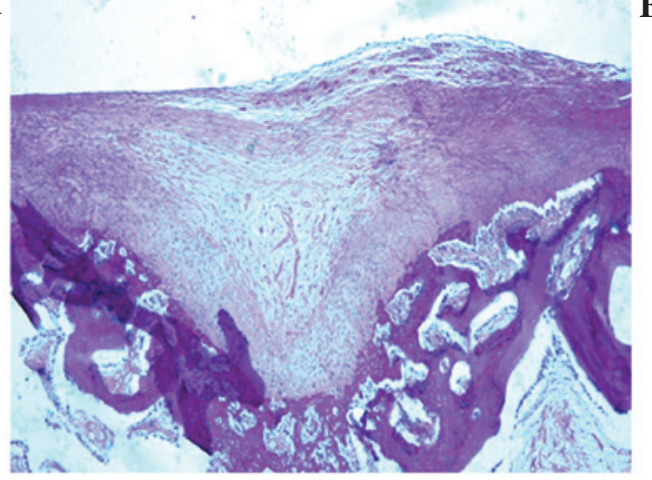

B

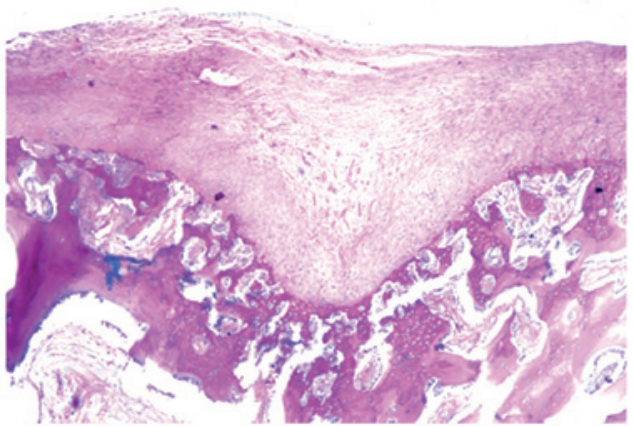

$\mathbf{C}$

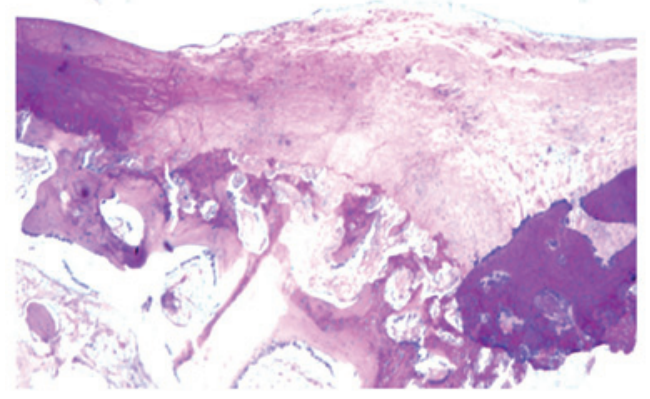

D

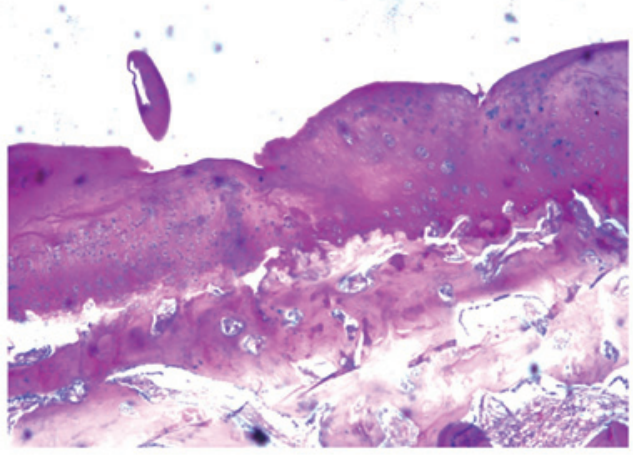

$\mathbf{E}$

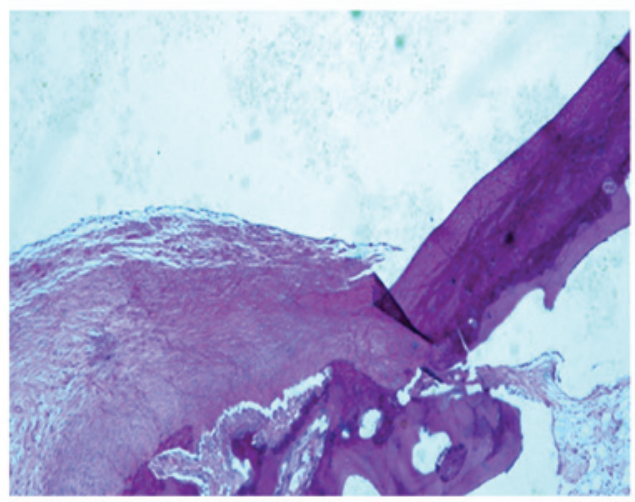

Figure 7. Histological staining revealing cartilage defects following implantation of BMSC scaffolds. The tissue samples were sectioned and stained with hematoxylin and eosin. (A) BMSCs cultured on scaffolds in the presence of PRP in a bioreactor. (B) BMSCs cultured on scaffolds without the use of the bioreactor. (C) BMSCs cultured in the bioreactor without PRP. (D) BMSCs cultured in the absence of the bioreactor or PRP. (E) Negative controls: Scaffold with no BMSCs. BMSCs, bone marrow stem cells; PRP, platelet-rich plasma.
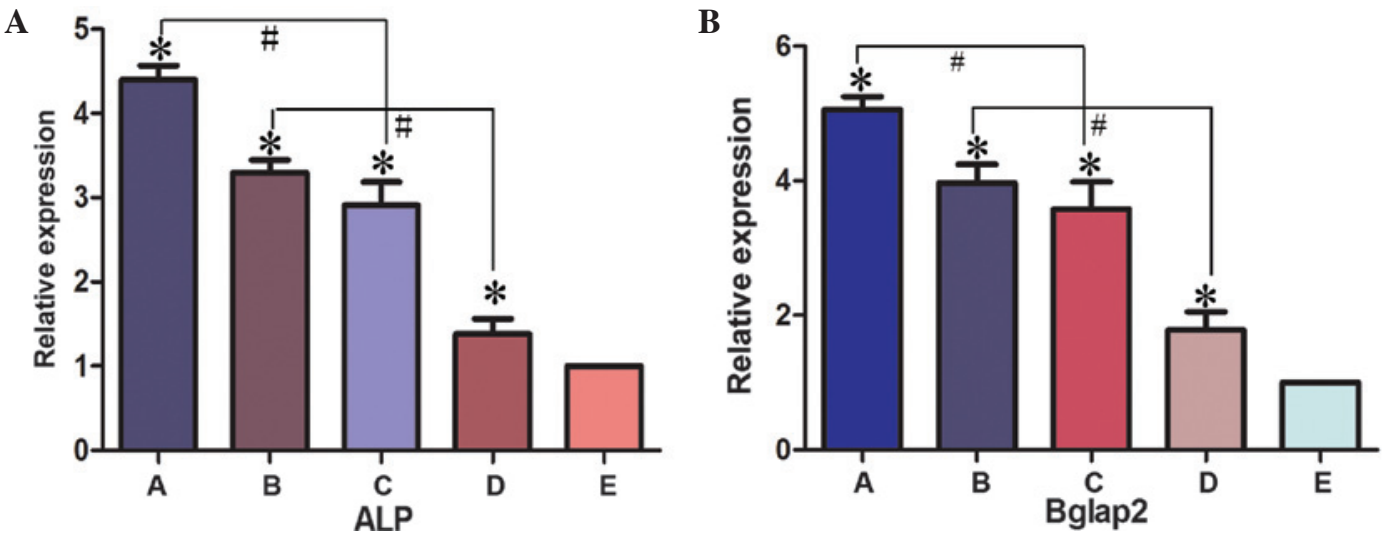

Figure 8. Gene expression levels of (A) ALP and (B) BGLAP2 were detected in the various experimental groups using reverse transcription-quantitative polymerase chain reaction. " $\mathrm{P}<0.05$ vs. control group (group E); " $\mathrm{P}<0.05$. Group A, BMSCs cultured with PRP in the bioreactor; Group B, BMSCs cultured with PRP without the use of the bioreactor; Group C, BMSCs cultured in the bioreactor without PRP; Group D, BMSCs cultured without PRP or use of the bioreactor; Group E, $\beta$-tricalcium phosphate scaffold only. ALP, alkaline phosphatase; BGLAP2, bone $\gamma$-carboxyglutamate protein 2; ALP, alkaline phosphatase; BGLAP2, bone $\gamma$-carboxyglutamate protein; BMSC, bone marrow stem cell; PRP, platelet-rich plasma. 
Consequently, a novel method for the efficient generation of tissue-engineered cells for the repair of bone defects has been developed in the present study.

\section{Discussion}

In the present study, we proposed that addition PRP and use of a bioreactor for the generation of BMSCs on scaffolds would enhance the healing process of osteochondral defects. Tissue-engineered bone was constructed by co-culturing BMSCs and $\beta$-TCP scaffolds in a 3D perfusion bioreactor. The present study revealed that BMSCs, PRP, $\beta$-TCP scaffolds and the use of a bioreactor had positive effects on bone formation and were able to enhance the healing of osteochondral defects.

In the present study, autologous PRP was used as a cytokine source to promote bone formation. PRP is a concentrate of platelets in a small volume of plasma. Once activated by an agonist, growth factors contained in platelets may be released and exert their biological functions. The initial clinical study using PRP for bone reconstruction therapy was performed by Marx et al (17). In this randomized study, 88 patients with mandibular defects were treated with autogenous cancellous bone grafts with or without the addition of activated PRP. Both the radiographic and the histomorphometric evaluation revealed a significantly greater percentage of bone generated in the presence of PRP (17). PRP therefore appears to exert a positive effect in the early phase of bone healing in combination with autogenous cancellous bone grafts (21-23), and in diabetic fracture healing (24). Other studies support the hypothesis that PRP has the ability to promote bone healing $(25,26)$ and strongly advocate its use in bone regenerative therapy $(27,28)$. The identification of the potential of PRP in the promotion of bone regeneration in recent years has generated a novel field of research in orthopedics. The effect of PRP in the treatment of periodontal intrabony defects in humans has also been investigated (29) and its beneficial effects in both periodontal and cosmetic surgical procedures have been suggested (30-32). In vitro studies have demonstrated the effects of platelet concentration on the proliferation and differentiation of primary osteoblasts and fibroblasts $(33,34)$. In two other in vivo studies $(35,36)$, goat PRP was prepared and activated with bovine thrombin and calcium chloride. PRP is currently used clinically in several countries and curative effects have been achieved (36). In addition, it has been demonstrated that PRP may enhance the healing of osteochondral defects and that the benefits may be detected at early stages of the healing process $(37,38)$. Froum et al (39) observed histomorphometric differences in bone regeneration among the samples of three surgical cases. Another study examining human PRP demonstrated upregulation of collagen synthesis in osteoblasts (39). Growth factors and BMSCs attract other progenitor cells or osteoclasts, and therefore initiate bone remodeling $(40,41)$. It is accepted that, subsequent to a bone injury, growth factors and host precursors are released (42). In addition, cell adhesion to extracellular matrices is essential in the development, maintenance and remodeling of osseous tissues $(42,43)$. The extracellular matrix component released by platelets is fibronectin, and the latter mediates adhesive interactions and has a central role in osteoblast survival, proliferation, differentiation and matrix mineralization, in addition to bone formation (44-46). The degranulation of PRP and release of growth factors may be achieved by the addition of thrombin in the presence of calcium chloride or by thawing the platelets (47). Studies have demonstrated that, in this combination, calcium sulfate acts as an activator of platelets and also as a delivery system for the platelet-released growth factors $(48,49)$. Therefore, it may be concluded that the endogenous growth factors released by platelets promote the healing process.

In a previous study performed by the authors (50), BMSCs were used as seed cells and $\beta$-TCP was used as a scaffold material, and a tissue-engineered osteochondral composite was successfully constructed in a perfusion bioreactor. This composite was used in the repair of osteochondral defects in beagles. It was found that certain grafts did not completely integrate into the areas of bone cartilage defects, indicating that the constructed osteochondral composite was not completely vascularized. In the present study, in the process of bone construction in the three-dimensional perfusion bioreactor, autologous PRP was used as a cytokine source to promote bone formation and vascularization. Autologous PRP is a convenient, economical and sustainable source of high-quality cytokines. The novel method for constructing tissue-engineered bone using BMSCs, $\beta$-TCP scaffolds and PRP can construct efficient tissue-engineered bone for cartilage repair.

A porous bio-ceramic 3D scaffold constructed of $\beta$-TCP was used in the present study for its favorable mechanical strength, high porosity ratio and ease of processing and molding. SEM displayed good adhesion and distribution of BMSCs on the $\beta$-TCP scaffold. Concurrently, extracellular matrix secretion was observed on the scaffold. Favorable cell proliferation and morphology was also detected using SEM, suggesting that the $\beta$-TCP scaffold has a high affinity for BMSCs. In brief, $\beta$-TCP scaffolds display several benefits in tissue engineering which as as follows: i) The $\beta$-TCP scaffold is able to provide a 3D space that may support BMSC growth, expose cells to shear force and improve unit expansion rate, thus promoting the differentiation of cartilage and bone; ii) a 3D structure may improve the biocompatibility of scaffolds and promote adhesion of BMSCs; iii) bone inducing growth factors will be gradually released by $\beta$-TCP in the process of cell culture due to the characteristics of the material. Overall, the $\beta$-TCP scaffold may be able to synchronously improve all three of the basic components required for bone and cartilage engineering. However, although the present study indicates that the $\beta$-TCP scaffold is an efficient biomaterial for BMSC adhesion and chondrogenic and osteogenic differentiation, further animal experiments are necessary to fully assess the scaffolds

The present study indicated that the use of a bioreactor and PRP was able to synergistically enhance cell proliferation and biosynthetic responses on scaffolds. Cell behavior is influenced by shear stress, tension and hydrostatic pressure stimulation. The continuous mechanical stimulation provided by the bioreactor, including shear stress stimulation, tension stimulation and hydrostatic pressure stimulation has effects on the morphology and function of BMSCs $(51,52)$. In the present study, BMSCs were cultured on a 3D scaffold in a perfusion bioreactor, a culture model that possesses several advantages 
compared with traditional planar 2D culture models. Firstly, it may provide mechanical stimulation to the BMSCs promoting their functionality. Secondly, the use of a bioreactor improves the distribution of nutrients within the scaffold. In addition, using the bioreactor provided a 3D growth environment, which promotes cell adhesion, growth and differentiation simultaneously, thereby reducing the effect of contact inhibition between the cells. Furthermore, the culture medium in a 3D bioreactor does not need to be replaced frequently, while the medium in a traditional 2D cell culture model requires intermittent replacement. The predominant advantage of the system is a result of the mechanical stimulation provided by the flowing fluid that may synergistically enhance cell proliferation and biosynthetic response on scaffolds, which is able to simulate a cell stress environment highly similar to that observed in vivo (51-53). In addition, cells may be fully combined with, or adhered to, the scaffold materials in the bioreactor, making cell culture closer to physiologic 3D growth $(54,55)$. The fluid shear stress provided by the bioreactor has a range of effects on cellular morphological and functional organization. It has previously been established that the fluid shear stress provided by a bioreactor is able to enhance the proliferation of MSCs (56). Notably, in the present study, it was revealed that PRP and the application of mechanical stimulation resulted in a greater number of cells and total collagen present on scaffolds after 21 days, compared with the use of PRP alone. The aforementioned findings suggested that mechanical stimulation is able to synergistically enhance cell proliferation and biosynthetic response on scaffolds. In addition, mechanical stimulation maintained the expression levels of ALP and BGLAP, the most important indicators of osteogenic differentiation (57). ALP has a critical role in the calcification process and has been used as a conventional marker of early osteoblast differentiation. BGLAP predominately appears in the mineralized formation as an indicator of osteoblast maturation. The expression levels of ALP and BGLAP therefore reflect the process of bone formation.

In conclusion, BMSCs, PRP, $\beta$-TCP scaffolds and the use of a bioreactor all had positive effects on bone formation. In the present study, a novel method for constructing tissue-engineered bone using BMSCs, $\beta$-TCP scaffolds and PRP was assessed. The tissue-engineered bone constructed using this method displayed active biologic characteristics in morphology and in function, and may therefore provide an improved approach for the repair of osteochondral defects.

The novel method for constructing tissue-engineered bone using BMSCs, $\beta$-TCP scaffolds and PRP detailed in the present study, will initiate the design and building of increasingly efficient tissue-engineered bone for cartilage repair.

\section{References}

1. Vacanti CA, Upton J: Tissue-engineered morphogenesis of cartilage and bone by means of cell transplantation using synthetic biodegradable polymer matrices. Clin Plast Surg 21: 445-62,1994

2. Liao J, Shi K, Ding Q, Qu Y, Luo F and Qian Z: Recent developments in scaffold-guided cartilage tissue regeneration. J Biomed Nanotechnol 10: 3085-3104, 2014.

3. Feng L, Wu H, E L, Wang D, Feng F, Dong Y, Liu H and Wang L: Effects of vascular endothelial growth factor 165 on bone tissue engineering. PLoS One 8: e82945, 2013.
4. Zou D, Zhang Z, Ye D, Tang A, Deng L, Han W, Zhao J, Wang S, Zhang W, Zhu C, et al: Repair of critical-sized rat calvarial defects using genetically engineered bone marrow-derived mesenchymal stem cells overexpressing hypoxia-inducible factor-1 $\alpha$. Stem Cells 29: 1380-1390, 2011.

5. Sun S, Ren Q, Wang D, Zhang L, Wu S and Sun XT: Repairing cartilage defects using chondrocyte and osteoblast composites developed using a bioreactor. Chin Med J (Engl) 124: 758-763, 2011.

6. Fridenshtein AI: Stromal bone marrow cells and the hematopoietic microenvironment. Arkh Patol 44: 3-11,1982 (In Russian).

7. Xue K, Qi L,Zhou G and Liu K: A two-step method of constructing mature cartilage using bone marrow-derived mesenchymal stem cells. Cells Tissues Organs 197: 484-495, 2013.

8. Intini G: The use of platelet-rich plasma in bone reconstruction therapy. Biomaterials 30: 4956-4966, 2009.

9. Fréchette JP, Martineau I and Gagnon G: Platelet-rich plasmas: Growth factor content and roles in wound healing. J Dent Res 84: 434-439, 2005

10. Lacoste E, Martineau I and Gagnon G: Platelet concentrates: Effects of calcium and thrombin on endothelial cell proliferation and growth factor release. J Periodontol 74: 1498-1507, 2003.

11. Hoffman R, Benz EJJ, Shattil SJ, Furie B, Cohen HJ, Silberstein LE, LE and McGlave P: Hematology: Basic principles and practice. Churchill Livingstone, Philadelphia, 2000.

12. Thiede MA, Smock SL, Petersen DN, Grasser WA, Thompson DD and Nishimoto SK: Presence of messenger ribonucleic acid encoding osteocalcin, a marker of bone turnover, in bone marrow megakaryocytes and peripheral blood platelets. Endocrinology 135: 929-937, 1994.

13. Landesberg R, Moses M and Karpatkin M. Risks of using platelet rich plasma gel. J Oral Maxillofac Surg 56: 1116-1117, 1998.

14. Xie L, Yu H, Deng Y, Yang W, Liao L and Long Q: Preparation, characterization and in vitro dissolution behavior of porous biphasic $\alpha / \beta$-tricalcium phosphate bioceramics. Mater Sci Eng C Mater Biol Appl 59: 1007-1015, 2016.

15. El-Fiqi A, Kim JH and Kim HW: Osteoinductive fibrous scaffolds of biopolymer/mesoporous bioactive glass nanocarriers with excellent bioactivity and long-term delivery of osteogenic drug. ACS Appl Mater Interfaces 7: 1140-1152, 2015.

16. Cheng L, Ye F, Yang R, Lu X, Shi Y, Li L, Fan H and Bu H: Osteoinduction of hydroxyapatite/beta-tricalcium phosphate bioceramics in mice with a fractured fibula. Acta Biomater 6: 1569-1574, 2010

17. Marx RE, Carlson ER, Eichstaedt RM, Schimmele SR, Strauss JE and Georgeff KR: Platelet-rich plasma: Growth factor enhancement for bone grafts. Oral Surg Oral Med Oral Pathol Oral Radiol Endod 85: 638-646, 1998.

18. Dwyer SD and Meyers KM: Anesthetics and anticoagulants used in the preparation of rat platelet-rich-plasma alter rat platelet aggregation. Thromb Res 42: 139-151, 1986.

19. Kasten P, Vogel J, Geiger F, Niemeyer P, Luginbühl R and Szalay K: The effect of platelet-rich plasma on healing in critical-size long-bone defects. Biomaterials 29: 3983-3992, 2008.

20. Livak KJ and Schmittgen TD: Analysis of relative gene expression data using real-time quantitative PCR and the $2^{-\Delta \Delta \mathrm{Ct}}$ method. Methods 25: 402-408, 2001.

21. Wiltfang J, Kloss FR, Kessler P, Nkenke E, Schultze-Mosgau S, Zimmermann R and Schlegel KA: Effects of platelet-rich plasma on bone healing in combination with autogenous bone and bone substitutes in critical-size defects. An animal experiment. Clin Oral Implants Res 15: 187-193, 2004.

22. Dugrillon A, Klüter H: Topical application of platelets for improved wound healing. Blood Ther Med 3: 21-26, 2002.

23. Fennis JP, Stoelinga PJ and Jansen JA: Mandibular reconstruction: A histological and histomorphometric study on the use of autogenous scaffolds, particulate cortico-cancellous bone grafts and platelet rich plasma in goats. Int J Oral Maxillofac Surg 33: 48-55, 2004.

24. Gandhi A, Dumas C, O'Connor JP, Parsons JR and Lin SS: The effects of local platelet rich plasma delivery on diabetic fracture healing. Bone 38: 540-546, 2006.

25. Kawase T, Okuda K, Wolff LF and Yoshie H: Platelet-rich plasma-derived fibrin clot formation stimulates collagen synthesis in periodontal ligament and osteoblastic cells in vitro. J Periodontol 74: 858-864, 2003.

26. Yamada Y, Ueda M, Naiki T, Takahashi M, Hata K and Nagasaka T: Autogenous injectable bone for regeneration with mesenchymal stem cells and plateletrich plasma: Tissue-engineered bone regeneration. Tissue Eng 10: 955-964, 2004. 
27. Marx RE: Platelet-rich plasma: Evidence to support its use. J Oral Maxillofac Surg 62: 489-496, 2004.

28. Freymiller EG and Aghaloo TL. Platelet-rich plasma: Ready or not? J Oral Maxillofac Surg 62: 484-488, 2004.

29. Ouyang XY and Qiao J: Effect of platelet-rich plasma in the treatment of periodontal intrabony defects in humans. Chin Med J (Engl) 119: 1511-1521, 2006.

30. Man D, PloskerH and Winland-Brown JE: The use of autologous platelet-rich plasma (platelet gel) and autologous platelet-poor plasma (fibrin glue) in cosmetic surgery. Plast Reconstr Surg 107: 229-237; discussion 238-239, 2001.

31. Mazor Z, Peleg M, Garg AK and Luboshitz J: Platelet-rich plasma for bone graft enhancement in sinus floor augmentation with simultaneous implant placement: Patient series study. Implant Dent 13: 65-72, 2004.

32. Kassolis JD, Rosen PS and Reynolds MA: Alveolar ridge and sinus augmentation utilizing platelet-rich plasma in combination with freeze-dried bone allograft: Case series. J Periodontol 71: $1654-1661,2000$

33. Choi BH, Zhu SJ, Kim BY, Huh JY, Lee SH and Jung JH: Effect of platelet-rich plasma (PRP) concentration on the viability and proliferation of alveolar bone cells: An in vitro study. Int J Oral Maxillofac Surg 34: 420-424, 2005.

34. Graziani F, Ivanovski S, Cei S, Ducci F, Tonetti M and Gabriele M The in vitro effect of different PRP concentrations on osteoblasts and fibroblasts. Clin Oral Implants Res 17: 212-219, 2006.

35. Fennis JP, Stoelinga PJ and Jansen JA: Mandibular reconstruction: A clinical and radiographic animal study on the use of autogenous scaffolds and platelet-rich plasma. Int J Oral Maxillofac Surg31: 281-286, 2002

36. Intini G, Andreana S, Intini FE, Buhite RJ and Bobek LA: Calcium sulfate and platelet-rich plasma make a novel osteoinductive biomaterial for bone regeneration. J Transl Med 5: 13 , 2007.

37. Cenni E, Perut F, Ciapetti G, Savarino L, Dallari D, Cenacchi A, Stagni C, Giunti A, Fornasari PM and Baldini N: In vitro evaluation of freeze-dried bone allografts combined with platelet rich plasma and human bone marrow stromal cells for tissue engineering. J Mater Sci Mater Med 20: 45-50, 2009.

38. Lieberman JR, Daluiski A and Einhorn TA: The role of growth factors in the repair of bone. Biology andclinical applications. J BoneJoint Surg Am 84: 1032-1044, 2002.

39. Froum SJ, Wallace SS, Tarnow DP and Cho SC: Effect of platelet-rich plasma on bone growth and osseointegration in human maxillary sinus grafts: Three bilateral case reports. Int J Periodontics Restorative Dent 22: 45-53, 2002.

40. Bruder SP, Kraus KH, Goldberg VM and Kadiyala S: The effect of implants loaded with autologous mesenchymal stem cells on the healing of canine segmental bone defects. J Bone Joint Surg Am 80: 985-996, 1998.

41. Roldán JC, Jepsen S, Miller J, Freitag S, Rueger DC, Açil Y and Terheyden H: Bone formation in the presence of platelet-rich plasma vs. bone morphogenetic protein-7. Bone 34: 80-90, 2004

42. Damsky $\mathrm{CH}$ : Extracellular matrix-integrin interactions in osteoblast function and tissue remodeling. Bone 25: 95-96, 1999.
43. Garcia AJ and Reyes CD: Bio-adhesive surfaces to promote osteoblast differentiation and bone formation. J Dent Res 84 : 407-413, 2005.

44. Weiss RE and Reddi AH: Role of fibronectin in collagenous matrix-induced mesenchymal cell proliferation and differentiation in vivo. Exp Cell Res 133: 247-254, 1981

45. Moursi AM, Damsky CH, Lull J, Zimmerman D, Doty SB, Aota $\mathrm{S}$ and Globus RK: Fibronectin regulates calvarial osteoblast differentiation. J Cell Sci 109: 1369-1380, 1996.

46. Zimmerman D, Jin F, Leboy P, Hardy S and Damsky C: Impaired bone formation in transgenic mice resulting from altered integrin function in osteoblasts. Dev Biol 220: 2-15, 2000.

47. Weibrich G, Gnoth SH, Otto M, Reichert TE and Wagner W: Growth stimulation of human osteoblast-like cells by thrombocyte concentrates in vitro. Mund Kiefer Gesichtschir 6 168-174, 2002 (In German).

48. Intini G, Andreana S, Margarone JE III, Bush PJ and Dziak R: Engineering a bioactive matrix by modifications of calcium sulfate. Tissue Eng 8: 997-1008, 2002.

49. Bateman J, Intini G, Margarone J III, Goodloe S III, Bush P, Lynch SE and Dziak R: Platelet-derived growth factor enhancement of two alloplastic bone matrices. J Periodontol 76: 1833-1841, 2005

50. Wang D, Jiang H, Wang S, Li H, Zhang H, Zhao L, Peng T, Cao Z and Sun S: Construction of tissue-engineered bone using a bioreactor and platelet-rich plasma. Exp Ther Med 8: 413-418, 2014.

51. Klein-Nulend J, van der Plas A, Semeins CM, Ajubi NE, Frangos JA, Nijweide PJ and Burger EH: Sensitivity of osteocytes to biomechanical stress in vitro. FASEB J 9: 441-445, 1995

52. Owan I, Burr DB, Turner CH, Qiu J, Tu Y, Onyia JE and Duncan RL: Mechanotransduction in bone: Osteoblasts are more responsive to fluid forces than mechanicalstrain. Am J Physiol 273: C810-C815, 1997.

53. Bakker AD, Soejima K, Klein-Nulend J and Burger EH: The production of nitric oxide and prostaglandin $\mathrm{E}(2)$ by primary bone cells is shear stress dependent. J Biomech 34: 671-677, 2001.

54. Wang Y, Kim UJ, Blasioli DJ, Kim HJ and Kaplan DL: In vitro cartilage tissue engineering with 3D porous aqueous-derived silk scaffolds and mesenchymal stem cells. Biomaterials 26 7082-7094, 2005.

55. Wang W, Itaka K, Ohba S, Nishiyama N, Chung UI, Yamasaki Y and Kataoka K: 3D spheroid culture system on micropatterned substrates for improved differentiation efficiency of multipotent mesenchymal stem cells. Biomaterials 30: 2705-2715, 2009.

56. Song K, Wang H, Zhang B, Lim M, Liu Y andLiu T: Numerical simulation of fluid field and in vitro three-dimensional fabrication of tissue-engineered bones in a rotating bioreactor and in vivo implantation for repairing segmental bone defects. Cell Stress Chaperones 18: 193-201, 2013.

57. Song Z, Wu C, Sun S, Li H, Wang D, Gong J and Yan Z: Quantitative analysis of factors influencing tissue-engineered bone formation by detecting the expression levels of alkaline phosphatase and bone $\gamma$-carboxyglutamate protein 2. Exp Ther Med 9: 1097-1102, 2015. 